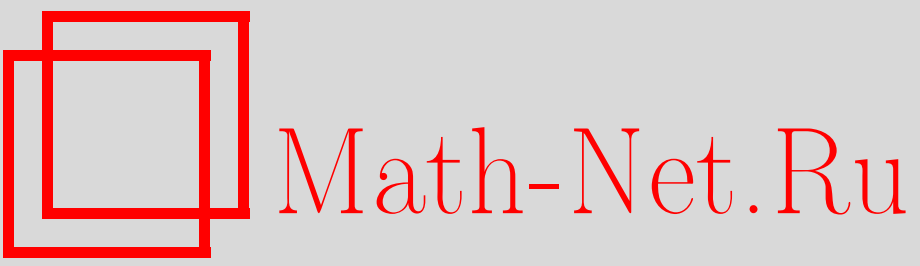

Д. В. Болотов, Гиперслоения неотрицательной кривизны Риччи, УМH, 2000, том 55, выпуск 2, 133-134

DOI: https://doi.org/10.4213/rm274

Использование Общероссийского математического портала Math-Net.Ru подразумевает, что вы прочитали и согласны с пользовательским соглашением

http://www.mathnet.ru/rus/agreement

Параметры загрузки:

IP : 54.210 .77 .194

26 апреля 2023 г., 13:34:59 


\title{
ГИПЕРСЛОЕНИЯ НЕОТРИЦАТЕЛЬНОЙ КРИВИЗНЫ РИЧЧИ
}

\author{
Д. В. Болотов
}

В данной работе мы предполагаем заданными многообразие и слоение на нем класса $C^{\infty}$. Гиперслоением мы будем называть слоение коразмерности 1. Гомологии и когомологии везде подразумеваются сингулярными с действительными коэффициентами, если иное не оговорено специально.

Если рассматривать трехмерную сферу со стандартной метрикой постоянной кривизны, то слоение Риба дает пример слоения, слои которого имеют неотрицательную секционную кривизну. В [1] ставится вопрос: существует ли на нечетномерной сфере размерности больше 3 гиперслоение со слоями неотрицательной секционной кривизны для какой-либо метрики на ней? Отметим, что на четномерной сфере не существует гиперслоения без особенностей. Препятствием является ненулевая эйлерова характеристика. Цель данной работы - доказать следующее утверждение.

Теорема А. Пусть на компактном многообразии $M^{2 n+1}$ задана риманова метрика положительной секционной кривизны. Тогда на нем не существует гиперслоения почти неотрицательной кривизнь Риччи.

(Многообразие имеет почти неотрицательную кривизну Риччи, если оно имеет неотрицательную кривизну Риччи вне некоторого компактного множества.)

Напомним некоторые резултаты, существенно применяемые при доказательстве.

Теорема 1 (Чигер, Громолл, [1]). Пусть $M$ - компактное многообразие неотрицательной кривизны Риччи, тогда существует компактное односвязное $(n-k)$-мерное многообразие $M_{0}(0 \leqslant k \leqslant n=\operatorname{dim} M)$ такое, что универсальная накрывающая $\widetilde{M}$ изометрична риманову произведению $M_{0} \times \mathbb{R}^{k}$ многообразия $M_{0}$ и евклидова фактора $\mathbb{R}^{k}$.

Теорема 2 [2]. Пусть $M$ - полное некомпактное многообразие почти неотрицательной кривизны Риччи. Тогда $M$ имеет конечное число концов.

Теорема (о стабильности, [3]). Пусть $F$ - трансверсально ориентируемое гиперслоение класса $C^{1}$ на компактном многообразии с компактным слоем $L$ таким, что $H^{1}(L, \mathbb{R})=0$, где $H^{1}(L, \mathbb{R})$ - первая группа когомологий с коэффициентами. Тогда все слои $F$ диффеоморфны $L$ и слоение $F$ есть расслоение над окружностью или отрезком со слоем L.

ТеОрема (о локальной стабильности, [3]). Пусть $F$ - слоение коразмерности $k$ u $L$ компактный слой $F$, тогда выполнено одно из следующих утверждений:

1) линейная голономия $L$ нетривиальна;

2) группа $H^{1}(L ; \mathbb{R})$ нетривиальна;

3) голономия слоя $L$ тривиальна и $L$ имеет трубчатую окрестность, в которой слоение является тривиальным расслоением.

ТеОрема 3 ([4], слабый вариант). Если $H_{1}(M, \mathbb{R})=0$ и $F$ имеет полиномиальный рост слоев, то всякое минимальное множсество F есть компактный слой.

Полиномиальньй рост слоев означает, что на каждом слое объем шара в каждой фиксированной точке полиномиальн зависит от радиуса.

Минимальное множество - это замкнутое насыщенное (т.е. состоящее из слоев) множество, не содержащее в себе такое же.

НАБРОСОК ДОКАЗАТЕЛЬСТВА ТЕОРЕМЫ А. Доказательство проведем от противного. Предположим, что существует гиперслоение $\mathcal{F}$ на $M^{2 n+1}$, удовлетворяющее условию теоремы. Заметим, что фундаментальная группа $M^{2 n+1}$ конечна [5]. Перейдя к универсальному накрытию, можно считать, что она - тривиальна. Поскольку кривизна Риччи слоев почти неотрицательна, слои имеют полиномиальный рост [5]. Из теоремы 3 следует, что $\mathcal{F}$ имеет компактный слой. Если этот слой - предельный для некомпактного, то он имеет неотрицателную кривизну Риччи. Согласно теореме 1 , надо рассмотреть три случая: 1) $k=0,2) k \geqslant 2,3) k=1$. 
Случай $k=0$. В этом случае фундаментальная группа слоев - конечна, поэтому из теоремы о стабильности следует, что слоение должно быть расслоением над окружностью (не надотрезком, так как $M^{2 n+1}$ - многообразие без края). Однако, если рассмотреть гомотопическую последовательность расслоения, видно, что фундаментальная группа окружности должна быть нулевой, что невозможно.

Случай $k \geqslant 2$. В этом случае в каждой точке $x$ слоя $L$ существовало бы 3 -мерное подпространство касательного пространства $T_{x} L$ такое, что секционная кривизна слоя $K_{L}$ была бы тождественным нулем на нем. Тогда можно было бы рассмотреть ограничение $\bar{\alpha}$ второй квадратичной формы $\alpha$ на это подпространство. Так как это подпространство трехмерное, то существовали бы главные кривизны $\lambda_{1}$ и $\lambda_{2}$ формы $\bar{\alpha}$ такие, что $\lambda_{1} \lambda_{2} \geqslant 0$. Пусть $e_{1}$ и $e_{2}-$ единичные векторы соответствующих главных направлений. Тогда по теореме Гаусса

$$
K_{e}\left(e_{1} \wedge e_{2}\right)=\lambda_{1} \lambda_{2},
$$

где $K_{e}$ - внешняя кривизна слоя, равная разности секционных кривизн слоя и объемлющего пространства.

В левой части - выражение меньше нуля по условию и выбору площадки, а в правой части больше или равное нулю в соответствии со сказанным выше. Это противоречие исключает случай $k \geqslant 2$.

Случай $k=1$. В этом случае, согласно теореме 1 , универсальная накрьвающая компактного слоя $L$ изометрична риманову произведению $\widetilde{M} \times \mathbb{R}$, где $\widetilde{M}$ - компактно. Нетрудно доказать, что $L$ имеет неотрицательную секционную кривизну. (Иначе мы попадаем в случай $k \geqslant 2$.) Значит, $L$ изометрично фактор-пространству $\widetilde{M} \times \mathbb{R} / G$, где $G$ дискретна и принадлежит Iso $(\widetilde{M} \times \mathbb{R})=$ Iso $\widetilde{M} \times$ Iso $\mathbb{R}[7]$. Верно следующее утверждение.

УТВеРЖДЕНИЕ 1. $H_{2 n-1}(L)=\mathbb{R}$ и существует компактная ориентируемая гиперповерхность $M \subset L$ с конечной фундаментальной группой такал, что класс $[M]$ является образующей в $H_{2 n-1}(L)$.

Пусть $L=A \cap B, A \cup B=M^{n+1}$, т.е. $A$ и $B$ - это подпространства, на которые слой $L$ разбивает $M^{2 n+1}$

Нетрудно показать, что $[M]$ как гомологический класс инъективно вкладывается хотя бы в одну из компонент $A$ или $B$. Пусть для определенности $[M]$ представляет ненулевой класс в $H_{2 n-1}(B)$.

Введем порядок на множестве компактных слоев. Пусть $\left\{L_{\alpha}\right\}$ - множество компактных слоев, a $A_{\alpha}, B_{\alpha}, M_{\alpha}$ - такие же, как $A, B, M$, и $B_{\alpha}$ принадлежит $B$ для всех $\alpha$. Скажем, что $L_{\gamma}<L_{\beta}$, если $B_{\beta} \subset B_{\gamma}$ и $\left[M_{\alpha}\right]$ является образующей в $H_{2 n-1}\left(B_{\alpha}\right)$ для всех $\alpha$.

УтВеРЖДЕнИЕ 2. Внутри В есть компактный слой $L_{1}$ maкой, что $L<L_{1}$.

Основная идея доказательства - показать, что некомпактный слой внутри компоненты $B$ обязан "наматываться" также и на другой компактный слой. Затем, используя теорему 2 , показьвается, что этот слой больше в смысле порядка, введенного выше.

Далее показывается, что бесконечная линейно упорядоченная последовательность компактных слоев должна вырождаться, что приводит к противоречию.

\section{СПИСОК ЛИТЕРАТУРЫ}

[1] Stuck G. // C. R. Acad. Sci. Ser. 1. 1991. V. 313. № 8. P. 519-522. [2] Liu Z.-D. // Proc. Sympos. Pure Math. 1993. V. 54. № 3. P. 459-465. [3] Thurston W. // Topology. 1974. V. 13. P. 347-352. [4] Plante Y. // Topology. 1973. V. 12. P. 177-181. [5] Бишоп Р., Криттенден Р. Геометрия многообразий. М.: Мир, 1967. [6] Дольд А. Лекции по алгебраической топологии. М.: Мир, 1976. [7] Cheeger J., Gromoll D. // Ann. of Math. 1972. V. 96. № 3. P. 413-443. 